\title{
Crecimiento y macronutrientes en Cenchrus purpureus cv. Taiwán con y sin fertilización en Veracruz
}

\section{Growth and macronutrients in Cenchrus purpureus cv. Taiwan with and without fertilization in Veracruz}

\begin{abstract}
Julio César Vinay-Vadillo ${ }^{1}$ (D)
Mónica Yazmín Herrera-Sotero 1 [D

Aguedo Juárez-Jiménez²

Maribel Montero-Lagunes (i)

Yuri Villegas-Aparicio2 2 (D)

Javier Francisco Enríquez-

Quiroz ${ }^{1 * \text { (D) }}$

Eduardo Daniel Bolaños-Aguilar ${ }^{3}$ (1) Sergio Iban Mendoza-Pedroza 4

${ }^{1}$ Instituto Nacional de Investigaciones Forestales, Agrícolas y Pecuarias, Campo Experimental La Posta. km 22.4 Carretera Federal Veracruz-Córdoba Paso del Toro, CP. 94277. Medellín, Veracruz, México. ${ }^{2}$ Instituto Tecnológico del Valle de Oaxaca. Ex-Hda. de Nazareno, CP. 71230. Santa Cruz Xoxocotlán, Oaxaca, México.

${ }^{3}$ INIFAP Campo Experimental Huimanguillo. Carretera Federal Humanguillocárdenas Kilómetro 1, CP. 86400 . Huimanguillo, Tabasco, México.

${ }^{4}$ Colegio de Postgraduados, Campus Montecillo. $\mathrm{km} 36.5$ Carretera Federal México-Texcoco, CP. 56230, Estado de México, México.

*Autor de correspondencia:

enriquez.javier@inifap.gob.mx
\end{abstract}

Artículo científico

Recibido: 03 de marzo 2021 Aceptado: 29 de septiembre 2021

Como citar: Vinay-Vadillo JC, HerreraSotero MY, Juárez-Jiménez A, MonteroLagunes M, Villegas-Aparicio Y, EnríquezQuiroz JF, Bolaños-Aguilar ED, MendozaPedroza SI (2021) Crecimiento y macronutrientes en Cenchrus purpureus cv. Taiwán con y sin fertilización en Veracruz. Ecosistemas y Recursos Agropecuarios Núm. Esp. II: e2942. DOI: 10.19136/era.a8nll.2942
RESUMEN. El objetivo fue determinar las curvas de crecimiento y tasas de nitrógeno, fósforo, potasio, así como su extracción y eficiencia del cultivar Taiwán con y sin fertilizar en Veracruz. El experimento se realizó durante la época de lluvias en clima $\mathrm{Aw}_{2}$, tropical sub-húmedo. Se establecieron siete parcelas de Cenchrus purpureus cv. Taiwán fertilizadas (141-43-20) y sin fertilizar. Los muestreos se realizaron cada 21 dias hasta los 168 días de edad de rebrote. A las muestras de forraje se les determinó Materia Seca (MS), N, P, K, Ca, Mg y S. Los datos se ajustaron a modelos lineales y no lineales. Se evaluaron con indicadores de bondad de ajuste para estimar los parámetros. Además, se calculó la eficiencia aparente del N, P, K. Los parámetros se analizaron en un diseño completamente al azar, con dos tratamientos, fertilizados (cuatro repeticiones) y sin fertilizar (tres repeticiones). Las medias se compararon con la prueba de Tukey con el software Minitab v17. Los parámetros del crecimiento, curvas de NPK, y extracción de NPK en el tratamiento fertilizado y no fertilizado demuestran, que para producir $6000 \mathrm{~kg} \mathrm{MS} \mathrm{ha}^{-1}$ a los 77 días de crecimiento, se requieren $186 \mathrm{~kg} \mathrm{~N}, 27 \mathrm{~kg} \mathrm{P}_{2} \mathrm{O}_{5}, 279 \mathrm{~kg} \mathrm{~K}{ }_{2} \mathrm{O}, 75 \mathrm{~kg} \mathrm{Ca}$, $22 \mathrm{~kg} \mathrm{Mg}$ y $3 \mathrm{~kg}$ de $\mathrm{S} \mathrm{ha}{ }^{-1}$. Mientras que sin fertilizar produjo $3000 \mathrm{~kg} \mathrm{MS} \mathrm{ha}^{-1}$, y extrajo una tercera parte de los nutrientes extraídos por el tratamiento fertilizado. Se concluye que las tasas de extracción de nutrientes fueron mayores en el tratamiento fertilizado.

Palabras clave: Extracción, eficiencia, NPK, materia seca, Gompertz.

ABSTRACT. The objective was to determine the growth curves and rates of nitrogen, phosphorus, potassium, as well as their extraction and efficiency from the cultivar Taiwan with and without fertilizing in Veracruz. The experiment carried out during the rainy season in $A w_{2}$, tropical sub-humid climate. Seven fertilized (14143-20) and unfertilized plots of Cenchrus purpureus cv Taiwan were established. The samplings carried out every 21 days until 168 days of regrowth age. The forage samples were determined Dry Matter (DM), N, P, K, Ca, Mg and S. The data adjusted to linear and non-linear models. They evaluated with goodness of fit indicators to estimate the parameters. In addition, the apparent efficiency of $\mathrm{N}, \mathrm{P}$, $\mathrm{K}$ was calculated. The parameters analyzed in a completely randomized design, with two treatments, fertilized (four repetitions) and unfertilized (three repetitions). The means compared with Tukey's test with Minitab v17 software. The growth parameters, NPK curves, and NPK extraction in the fertilized and unfertilized treatment show that to produce $6000 \mathrm{~kg} \mathrm{DM} \mathrm{ha}^{-1}$ at 77 days of growth, $186 \mathrm{~kg} \mathrm{~N}$, $27 \mathrm{~kg} \mathrm{P}_{2} \mathrm{O}_{5}, 279 \mathrm{~kg} \mathrm{~K}_{2} \mathrm{O}, 75 \mathrm{~kg} \mathrm{Ca}, 22 \mathrm{~kg} \mathrm{Mg}$ and $3 \mathrm{~kg}$ of $\mathrm{S} \mathrm{ha}{ }^{-1}$ are required. While without fertilizing, it produced $3000 \mathrm{~kg} \mathrm{DM} \mathrm{ha}^{-1}$, and extracted a third of the nutrients extracted by the fertilized treatment. It concluded that the nutrient extraction rates were higher in the fertilized treatment.

Key words: Extraction, efficiency, NPK, Dry mater, Gompertz. 


\section{INTRODUCCIÓN}

En el trópico mexicano, uno de los principales problemas del ganado en pastoreo es la subalimentación debido a la baja disponibilidad y calidad del forraje en la época seca. En esta región, la producción de leche y carne bovina se basa fundamentalmente en el pastoreo extensivo, donde la producción y calidad del forraje juega un papel importante sobre la productividad animal, ya que, es la principal fuente de energía y proteína (Ortega et al. 2009). Es por esto que, diversos factores afectan la producción y calidad del forraje; la especie, variedad, estado fenológico al momento de la cosecha, fertilidad del suelo, prácticas agronómicas de fertilización, riego, y factores climáticos (temperatura ambiental, precipitación pluvial, duración del día), plagas y enfermedades, determinan variaciones estacionales en la producción y calidad nutricional (Ortega et al. 2009). La especie Cenchrus purpureus Syn de Pennisetum purpureum, es un pasto perenne $\mathrm{C} 4$, de origen africano, la cual ha sido naturalizada en la mayoría de los países tropicales del mundo, y se utiliza para la alimentación animal (Negawo et al. 2017), por sus caracteristicas de alto rendimiento de materia seca, excelente palatabilidad y calidad del forraje, bajo un manejo adecuado. Pero requiere altas necesidades de macronutrientes, dado su alto rendimiento, maduración rápida y particularmente si se utiliza bajo corte y acarreo (Cook et al. 2020). La especie Cenchrus purpureus fue introducida en 1950 al Campo Cotaxtla en el estado de Veracruz, donde fue evaluada junto a una amplia colección de gramíneas (Ramos-Sánchez 1985). El zacate Taiwán ha sido ampliamente aceptado por los productores debido a su buen desempeño en adaptación al ambiente y alta capacidad para producir forraje, sin embargo, requiere altas dosis de fertilización, para alcanzar rendimientos aproximados a 50 t MS ha $^{-1}$ año $^{-1}$ (Chandler et al. 1983, CalzadaMarín et al. 2014, Rueda et al. 2016, Reyes-Castro et al. 2018). Actualmente, se utilizan herramientas que ayudan a evaluar el rendimiento, necesidades de nutrientes y el desempeño de los forrajes en el trópico mexicano; las curvas y tasas crecimiento, curvas de extracción y concentración critica de nutrientes como el nitrógeno $(\mathrm{N})$, fósforo $(\mathrm{P})$ y potasio $(\mathrm{K})$ y la eficiencia de cada uno de ellos (López-Astilleros et al. 2020).

El crecimiento de las plantas, ha sido descrito por una gran cantidad de modelos matemáticos, entre ellos el más empleado es el desarrollado por Gompertz (Turner et al. 1976, France y Thornley 1984, Raji et al. 2014). que permite estimar o predecir su comportamiento productivo en diferentes condiciones (Thornley y France 2007). Al respecto, Winsor (1932) y Rodríguez et al. (2011) propusieron utilizarlo para la descripción de fenómenos biológicos asociados al crecimiento. No obstante, para su correcta utilización se deben tener en cuenta tres aspectos básicos: a) Bondad de ajuste, b) Capacidad de interpretación biológica, y c) Exigencias computacionales (Chacín 1998). Este modelo asume que la velocidad de crecimiento se incrementa hasta que alcanza un máximo y finalmente una forma asintótica (Lawrence y Fowler 2002). La correspondiente curva es sigmoidea, con un punto de inflexión (máxima tasa de crecimiento con la edad) y una asíntota (Lawrence y Fowler 2002). Las curvas de extracción o absorción de nutrientes, son una representación gráfica y representan las cantidades de cada elemento extraído por la planta durante su ciclo de vida y depende básicamente del potencial genético de la planta, la edad, las condiciones del clima; temperatura, humedad, e intensidad solar. Conociendo las curvas de extracción o absorción, se determina el tiempo donde ocurre la mayor extracción, a su vez, permite definir el tiempo de aplicación de los fertilizantes o la generación de un programa de fertilización adecuado, para conocer la eficiencia de los fertilizantes (López-Astilleros et al. 2020). También, ayuda a determinar las necesidades de elementos nutritivos para la producción de biomasa (Rueda et al. 2016, Ventura et al. 2017). En Cenchurs purpureus las tasas de extracción de nutrientes son elevadas, ya que para una producción de $28200 \mathrm{~kg} \mathrm{MS} \mathrm{ha}^{-1}$ año ${ }^{-1}$, extrae $338(\mathrm{~N}), 72(\mathrm{P}), 564(\mathrm{~K}), 108(\mathrm{Ca})$ y 71 (Mg) kg ha ${ }^{-1}$ año $^{-1}$ (Chandler et al. 1983). Sin embargo, existen bases teóricas y experimentales en varios cultivos, donde se menciona que la estimación de la concentración crítica de nitrógeno, fósforo y 
potasio a través de modelos no lineales que permitan optimizar el uso de fertilizantes y ajustar los excesos o deficiencias, incrementando la producción de biomasa de manera que se eviten daños al ambiente (Flénet et al. 2006, Ziadi et al. 2008, Agnusdei et al. 2010). La optimización de los macronutrientes durante el crecimiento, es de fundamental importancia en la producción de forrajes en el trópico, ya que, no es suficiente conocer su contenido en el suelo, para establecer los requerimientos de los forrajes y así, obtener un potencial productivo elevado (Fagundes et al. 2006). Consecuentemente el conocimiento de la eficiencia del uso de macronutrientes como NPK (NUE), eficiencia de la absorción y conversión de NPK (NUpE y NCE, respectivamente) en los forrajes, contribuyen a determinar los requerimientos y las estrategias de fertilización (Marino et al. 2004). Estos han sido estudiados ampliamente por Isfan (1993), Ortiz-Monasterio et al. (1997), Wilkins et al. (1997), Dreccer et al. (2000) y Marino et al. (2004), quienes reportan que los nutrimientos requeridos para lograr un crecimiento elevado, se relaciona con el genotipo. Por lo anterior, el objetivo fue determinar los parámetros de las curvas de nitrógeno, fósforo y potasio, curvas de extracción de macronutrientes y la estimación de la eficiencia del uso, absorción y conversión del Nitrógeno, Fósforo y Potasio en la Llanura costera Veracruzana, con el fin de conocer la dinámica de los macronutrientes que requiere el cultivar Taiwán durante su crecimiento.

\section{MATERIALES Y MÉTODOS}

El estudio se llevó a cabo de julio de 2010 a enero de 2011, durante la época de lluvias, en el Campo Experimental La Posta de Paso del Toro, Veracruz, localizado en las coordenadas $19^{\circ} 00^{\prime} 49^{\prime \prime}$ LN y $96^{\circ} 08^{\prime} 19^{\prime \prime}$ LO a 12 msnm (INEGI 2009). El clima predominante en el área corresponde al $\mathrm{Aw}_{2}$ tropical sub-húmedo (Vidal 2005), con precipitación pluvial de $1461 \mathrm{~mm}$, humedad relativa de $77.4 \%$, temperatura media de $25^{\circ} \mathrm{C}$, máxima de $35^{\circ} \mathrm{C}$ y mínima de $15{ }^{\circ} \mathrm{C}$. Los suelos predominantes son del tipo vertisol, pH ácido (4.9-5.4), textura de migajón arcilloarenosa, un contenido mediano de materia orgánica y nitrógeno inorgánico de entre 1.6-2.6\% y 7-21 ppm, respectivamente; fósforo de $17.6-78.5 \mathrm{ppm}$, potasio de 174 ppm, calcio de 1590-1936 ppm, y magnesio de 288-418 ppm.

\section{Diseño experimental}

Siete parcelas de pasto Taiwán se establecieron al azar con medidas repetidas en el tiempo, con cuatro repeticiones con fertilización nitrogenada, fosfatada y potásica y tres repeticiones sin fertilización. En las parcelas fertilizadas, el primer corte se realizó a 14 días de crecimiento y después cada 21 días hasta los 161 días de edad de rebrote, en las parcelas sin fertilizar cada 21 días hasta 168 días de edad de rebrote.

\section{Manejo agronómico}

El terreno fue preparado con subsoleo, barbecho, doble rastra y surcado a $80 \mathrm{~cm}$. Se usaron dos esquejes o estacas de tres nudos, los cuales se sembraron cada $80 \mathrm{~cm}$ sobre el terraplén del surco, procurando que dos de los tres nudos quedaran enterrados en un ángulo de $45^{\circ}$ con relación a la superficie. El periodo de establecimiento fue durante 2009. El área de muestreo en cada fecha de corte fue de $5.76 \mathrm{~m}^{2}$, equivalente a tres surcos, $2.4 \mathrm{~m}$ de ancho, por 2.4 $\mathrm{m}$ de largo, se realizaron un total de ocho cortes. Al inicio del experimento se realizó un corte de uniformidad a $25 \mathrm{~cm}$, cuando la planta tenía una edad de 12 meses. Se realizó una fertilización con una dosis de 141-43-20; 200 kg de Urea (46-00-00), 50 kg de Fosfato Diamónico (18-46-00) y $200 \mathrm{~kg}$ de la mezcla (20-10-10), la cual se aplicó en dos ocasiones; a los 8 y 60 días después del corte de uniformidad. El forraje (materia verde) se cosechó a una altura de $25 \mathrm{~cm}$ del suelo, y cada 21 días de crecimiento. Durante los muestreos se registró la producción de materia seca (MS) de la planta completa de cada parcela, de la cual se tomó una submuestra de la planta completa (300 g) para secarla a $55^{\circ} \mathrm{C}$ hasta alcanzar el peso constante utilizando una estufa de aire forzado.

\section{Análisis químico}

El contenido de nitrógeno total en la submuestra se determinó por el método MacroKjeldhal en 
muestras de $0.5 \mathrm{~g}$ por triplicado de planta completa; fósforo con Bray $\mathrm{P} 1$ vanadato de amonio en espectrofotómetro UV-Visible (UV/VIS Lambda $2^{\circledR}$, Perkin Elmer $^{\circledR}$, USA). Potasio, calcio y magnesio por absorción atómica y azufre por el método turbidimétrico con sulfato de bario en espectrofotómetro UV-Visible.

\section{Curva y tasa de crecimiento}

Se procedió a realizar un análisis de regresión no lineal empleando el modelo Gompertz, aplicando el algoritmo de Powell como el método de minimización con los resultados del rendimiento de biomasa en $\mathrm{kg} \mathrm{MS} \mathrm{ha}^{-1}$, en cada tiempo de muestreo. Para ello se utilizó el software Micromath Scientist ${ }^{\circledR}$, donde la función de Gompertz utilizada fue: (Horgan 2005)

$$
Y=A \exp ^{-\exp (-\mu(x-B))}
$$

Dónde: $Y=$ Rendimiento de forraje $(\mathrm{kg} \mathrm{MS}$ ha $^{-1}$ ); $\mathrm{X}=$ Tiempo (días); $\mathrm{A}=$ Máxima producción biomasa ( $\mathrm{kg} \mathrm{MS} \mathrm{ha}^{-1}$ ); $\mathrm{B}=$ Punto de inflexión, que indica el fin del crecimiento e inicia el decrecimiento (días) y $\mu=$ Velocidad del crecimiento $\left(\mathrm{kg} \mathrm{MS} \mathrm{ha}^{-1}\right.$ día $\left.{ }^{-1}\right)$.

Los indicadores de bondad de ajuste, que se emplearon para el modelo fueron: el coeficiente de determinación y correlación, y el criterio selección de modelo (MSC siglas en inglés), que indica que a medida que el valor es mayor, el modelo tiene mejor ajuste. Para mejorar el ajuste se utilizó un coeficiente de ponderación en cada análisis. La tasa de crecimiento se calculó en $\mathrm{kg} \mathrm{MS} \mathrm{ha}{ }^{-1} \mathrm{~d}^{-1}$, con los datos de producción de MS estimados con el modelo de Gompertz en las edades del forraje, con la siguiente ecuación (Ramírez et al. 2011):

$$
T C=\frac{M S P_{t 2}-M S P_{t 1}}{T 2-T 1}
$$

Dónde: $\mathrm{TC}=$ Tasa de crecimiento $\left(\mathrm{kg} \mathrm{MS} \mathrm{ha}^{-1}\right.$ $\left.\mathrm{d}^{-1}\right), \mathrm{MSP}_{t 2}=$ Materia Seca presente en el tiempo dos $\left(\mathrm{kg} \mathrm{MS} \mathrm{ha}^{-1}\right), \mathrm{MSP}_{t 1}=$ Materia seca presente en el tiempo uno $\left(\mathrm{kg} \mathrm{MS} \mathrm{ha}^{-1}\right), \mathrm{T} 2$ = tiempo final, $\mathrm{T} 1=$ tiempo inicial.

\section{Curvas de Nitrógeno, Fósforo y Potasio}

Se determinó el contenido de nitrógeno $(\mathrm{N})$, fósforo $(P)$ y potasio $(K)$ de las muestras de forraje de planta completa a dos repeticiones, durante el crecimiento en los muestreos establecidos previamente. Con los datos del porcentaje de NPK en base seca y la producción de biomasa, se determinaron los parámetros $M_{1}$ y $M_{2}$ con la siguiente ecuación de potencia (Justes et al. 1994):

$$
Y=M_{1} X^{-M 2}
$$

Dónde: $Y=$ es el porcentaje en base seca de NPK, $X=$ en la producción de biomasa (t de MS $\left.\mathrm{ha}^{-1}\right), \mathrm{M}_{1}=$ Valor crítico del componente $\left(\mathrm{g} 100 \mathrm{~g}^{-1}\right.$ MS) para producir una tonelada de MS en su máximo crecimiento; $\mathrm{M}_{2}=$ Es la disminución de la concentración de cada componente durante el crecimiento.

En el ajuste de los datos se utilizó el método de minimización de Levenberg-Marquart (Software Kaleidagraph $\mathrm{v} 3.0^{\circledR}$ ). Los indicadores de bondad de ajuste fueron, el coeficiente de correlación y la Chi cuadrada, este último, cuando es menor, el ajuste de los datos es mejor.

\section{Curvas de extracción de Nitrógeno (N), Fósforo $(\mathrm{P})$, Potasio $(\mathrm{K})$, Calcio $(\mathrm{Ca})$, Magnesio $(\mathrm{Mg})$ y Azufre (S)}

La extracción de nutrientes $\left(\mathrm{kg} \mathrm{ha}^{-1}\right)$, en las parcelas fertilizadas y sin fertilizar, se determinaron mediante la composición nutrimental de N, P, K, Ca, $\mathrm{Mg}$ y $\mathrm{S}$ en cada tiempo, multiplicadas por la producción de biomasa estimados con las curvas de crecimiento y se ajustaron con un polinomio de orden cuadrático.

\section{Eficiencia del Uso, Absorción y Conversión de NPK}

Se calculó mediante lo establecido por Marino et al. (2004). Para determinar la eficiencia de uso se determinó con la siguiente fórmula: Eficiencia del uso aparente del NPK (NPKUE) $=[$ Biomasa de parcela fertilizada - Biomasa de parcela no fertilizada (kg MS ha ${ }^{-1}$ )]/ Nitrógeno aplicado (kg NPK ha ${ }^{-1}$ ). Mientras que la eficiencia de absorción se determinó como: Eficiencia de la Absorción aparente de NPK 
$(\mathrm{NPKUpE})=[$ Absorción de NPK parcela fertilizada - Absorción de NPK parcela no fertilizada (kg NPK $\left.\mathrm{ha}^{-1}\right)$ ]/ Nitrógeno aplicado ( $\mathrm{kg} \mathrm{NPK} \mathrm{ha}^{-1}$ ). En lo que corresponde a la eficiencia de conversión aparente se determinó como: Eficiencia de la Conversión aparente del NPK (NPKCE) $=$ [Biomasa de parcela fertilizada - Biomasa de parcela no fertilizada ( $\mathrm{kg}$ MS $\mathrm{ha}^{-1}$ ) / [Absorción de NPK parcela fertilizada - Absorción de NPK parcela no fertilizada (kg NPK ha-1)].

\section{Diseño Experimental y Análisis Estadístico}

Los parámetros obtenidos, así como el coeficiente de bondad ajuste, fueron analizados con un ANOVA bajo un diseño completamente al azar para los tratamientos sin y con la aplicación de fertilizante NPK. Los datos se analizaron con el software MINITAB versión 17 en procedimiento GLM. La comparación de medias se realizó con la prueba de Tukey a una alfa de $0.05 \%$.

\section{RESULTADOS}

En la Figura 1, se observa el comportamiento de la curva sigmoidal del crecimiento del cultivar Taiwán fertilizado y exponencial para el tratamiento sin fertilizar. Los residuales obtenidos del ajuste, muestran en ambos casos, una distribución normal de los datos, indicando que el modelo utilizado fue el adecuado. Se encontró que la tasa de crecimiento tiene una distribución de un polinomio cúbico, que para el caso del forraje fertilizado mostró la ecuación $Y=$ $26.7+1.36 \mathrm{X}-0.010 \mathrm{X}^{2}+0.0000197 \mathrm{X}^{3}$ y para el forraje sin fertilizar $Y=27.7+0.92 X-0.011 X^{2}+0.0000343 X^{3}$, del cual se muestran los datos estimados.

La media de los parámetros de la curva de crecimiento del cultivar Taiwán con y sin fertilización y sus medidas de dispersión, se presentan en la Tabla 1. La producción máxima de biomasa, expresado por el parámetro A fue de $11068 \mathrm{~kg} \mathrm{MS}$ ha $^{-1}$ a los 161 días de crecimiento, fertilizado con una dosis de 141-43-20, que fue diferente $(P \leq$ 0.05) en relación a la baja producción (3 $961 \mathrm{~kg}$ $\mathrm{MS} \mathrm{ha}^{-1}$ ) del forraje que no fue fertilizado a los 168 días de crecimiento, que dependió únicamente de los nutrientes disponibles en el suelo, considerado moderadamente ácido y contenido mediano de materia orgánica. Aunque, el forraje no fertilizado creció más rápido, no logro mayor producción de biomasa debido a la baja disponibilidad de nutrientes, propiciando que el punto de inflexión (B), es decir el punto en que el forraje llego a su máximo crecimiento y empieza a decrecer, fuera menor (34.6 días) al observado en el forraje fertilizado (54.4 días), sin embargo, no se encontraron diferencias estadísticas significativas $(P \geq 0.05)$. La velocidad de crecimiento $(\mu)$ en el forraje fertilizado fue menor $\left(25.4 \mathrm{~kg} \mathrm{MS} \mathrm{ha}^{-1} \mathrm{día}^{-1}\right.$ ) que el observado en el forraje sin fertilizar $(31.8 \mathrm{~kg}$ MS ha ${ }^{-1}$ día $\left.^{-1}\right)$, sin presentar diferencias estadísticas significativa $(P \geq 0.05)$.

Los valores de la bondad de ajuste de la curva y tasa de crecimiento se muestran en la Tabla 2, ambos casos, donde se empleó el modelo no lineal y lineal. Los valores de los coeficientes $R^{2}, R, y C$ son cercanos a uno (0.99) y no presentaron diferencias $(P \geq 0.05)$ en los tratamientos. Para el caso del criterio de selección del modelo (MSC), el valor para el modelo de Gompertz con fertilización fue mayor (3.89) y diferente $(P \leq 0.05)$ al forraje sin fertilizar (2.36), sin embargo, resulta interesante resaltar que el comportamiento es diferente cuando se aplica el modelo lineal en la tasa de crecimiento, debido a que el mejor ajuste se encuentra con los datos del forraje sin fertilizar (3.49). Cabe mencionar que los valores de MSC en ambos modelos, se consideran como un indicador del buen ajuste de los datos entre los valores observados y estimados.

Los comportamientos de los datos no lineales se presentan en la Figura 2, donde se observa el porcentaje de NPK con respecto a la producción de biomasa, ajustados con la ecuación de potencia, mostrando una tendencia a la disminución del contenido de NPK con respecto a la biomasa (t MS ha ${ }^{-1}$ ) en el forraje fertilizado y sin fertilizar. Cabe mencionar, que los porcentajes de NPK en los forrajes fertilizados inician con valores más elevados que los forrajes sin fertilizar. Con la aplicación de la ecuación de potencia se estimaron los parámetros de la concentración crítica $\left(M_{1}\right)$ y del coeficiente de disminución $\left(\mathrm{M}_{2}\right)$ de los nutrientes NPK del cultivar Taiwán, con y sin fertilización, el cual se pre- 

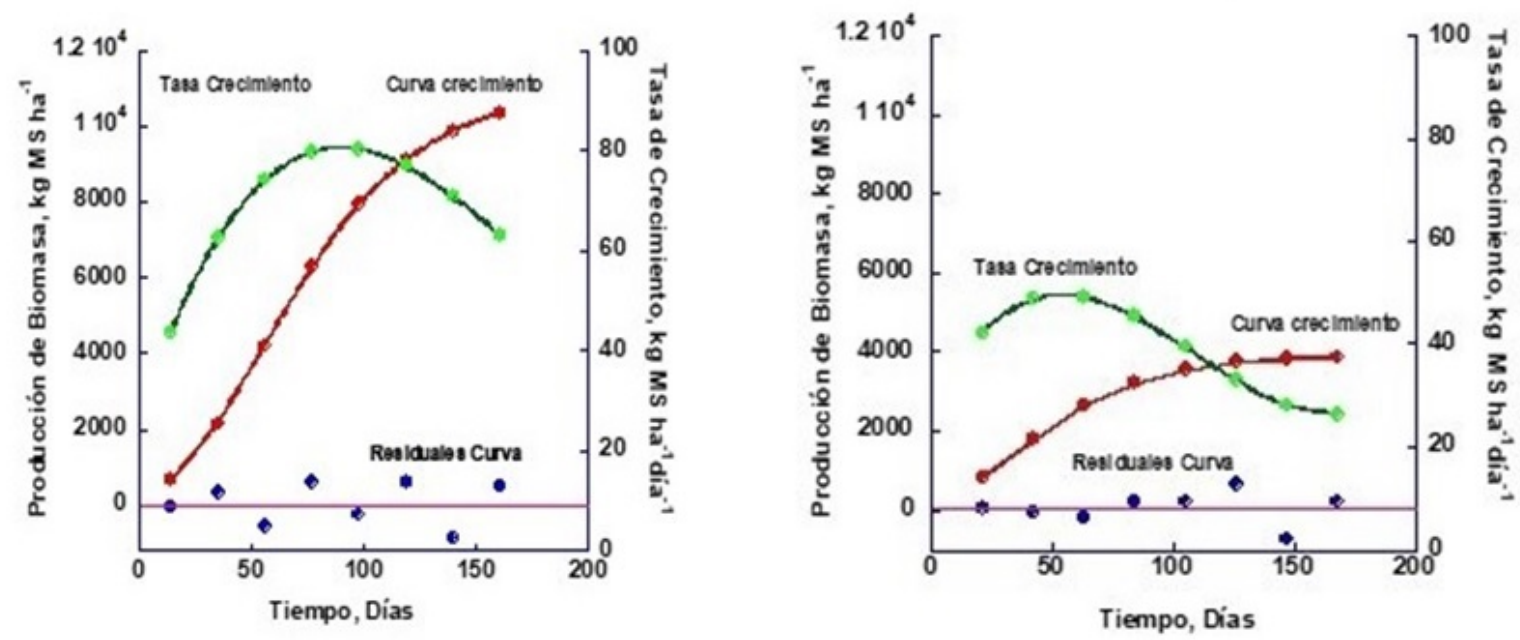

Figura 1. Datos ajustados de la curva y tasa de crecimiento del Cenchrus pupureus cultivar Taiwán: a) Fertilizado y b) Sin fertilizar.

Tabla 1. Parámetros de la curva de crecimiento del cultivar Taiwán con y sin fertilización, con ajuste del modelo de Gompertz.

\begin{tabular}{lccccc}
\hline Cultivar Taiwán & Parámetro & Media & $\begin{array}{c}\text { Desviación } \\
\text { estándar }\end{array}$ & $\begin{array}{c}\text { Límite } \\
\text { inferior }\end{array}$ & $\begin{array}{c}\text { Límite } \\
\text { superior }\end{array}$ \\
\hline Fertilizado $(4)^{\star}$ & $\mathrm{A}$ & $11,068^{a}$ & 925 & 8690 & 13446 \\
& $\mathrm{~B}$ & $54.4^{a}$ & 5.5 & 40.3 & 68.4 \\
Sin fertilizar (3) & $\mu$ & $25.4^{a}$ & 3.9 & 15.2 & 35.7 \\
& $\mathrm{~A}$ & $3,961^{b}$ & 369 & 3011 & 4910 \\
& $\mathrm{~B}$ & $34.6^{a}$ & 5.3 & 21.1 & 48.2 \\
& $\mu$ & $31.8^{a}$ & 9.3 & 8.1 & 55.6 \\
\hline
\end{tabular}

${ }^{a b}$ Literales diferentes de los parámetros de la curva de crecimiento entre el forraje fertilizado y sin fertilizar significancia estadística $(P<0.05)$, prueba de medias Tukey $=0.05 . \mathrm{A}=$ Madurez o producción máxima $\left(\mathrm{kg} \mathrm{MS} \mathrm{ha}^{-1}\right) ; \mathrm{B}=$ Punto de inflexión o cambio de crecimiento a decrecimiento (días); $\mu$ = velocidad de crecimiento (kg MS $\mathrm{ha}^{-1}$ día $\left.^{-1}\right)$. *Número de repeticiones.

Tabla 2. Indicadores de bondad de ajuste de la curva y tasa de crecimiento del cultivar Taiwán con o sin fertilizar.

\begin{tabular}{|c|c|c|c|c|c|}
\hline \multirow[b]{2}{*}{ Indicador } & \multicolumn{2}{|c|}{$\begin{array}{c}\text { Curva, Modelo No lineal, } \\
\text { Gompertz }\end{array}$} & \multirow[b]{2}{*}{ Indicador } & \multicolumn{2}{|c|}{$\begin{array}{l}\text { Tasa, Modelo Lineal, } \\
\text { Polinomio cúbico }\end{array}$} \\
\hline & $\begin{array}{l}\text { Fertilizado } \\
(4)^{\star}\end{array}$ & $\begin{array}{l}\text { Sin Fertilizar } \\
(3)^{*}\end{array}$ & & $\begin{array}{l}\text { Fertilizado } \\
(4)^{\star}\end{array}$ & $\begin{array}{l}\text { Sin Fertilizar } \\
(3)^{*}\end{array}$ \\
\hline $\mathrm{R}^{2}$ & $0.99^{a}$ & $0.98^{a}$ & $R^{2}$ & $0.99^{a}$ & $0.99^{a}$ \\
\hline $\mathrm{R}$ & $0.99^{a}$ & $0.95^{a}$ & $\mathrm{R}$ & $0.97^{a}$ & $0.98^{a}$ \\
\hline C & $0.99^{a}$ & $0.97^{a}$ & C & $0.98^{a}$ & $0.99^{a}$ \\
\hline MSC & $3.89^{a}$ & $2.36^{b}$ & MSC & $2.85^{b}$ & $3.49^{a}$ \\
\hline
\end{tabular}

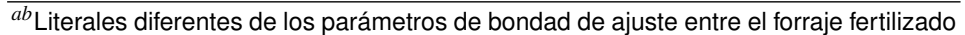
y $\sin$ fertilizar significancia estadística $(P<0.05)$, prueba de medias Tukey $=0.05 . R^{2}$ = Coeficiente de determinación; $\mathrm{R}=$ Coeficiente de correlación; $\mathrm{C}=$ Correlación; MSC $=$ Criterio de selección del modelo. $\left({ }^{*}\right)=$ Repeticiones.

senta en la Tabla 3. Los resultados del cálculo de aproximación de las necesidades de NPK $\left(\mathrm{kg} \mathrm{ha}^{-1}\right)$, empleando el parámetro $M_{1}$ y la tasa máxima de crecimiento de $79 \mathrm{~kg} \mathrm{ha}^{-1}$ día $^{-1}$, logrado en 77 días en el Taiwán fertilizado y de $49 \mathrm{~kg} \mathrm{ha}^{-1}$ día $^{-1}$ en
63 días en el Taiwán sin fertilizar. En la Tabla 4, se presentan los valores correspondientes a los indicadores de bondad de ajuste, resultado de la aplicación de la ecuación de potencia en la determinación de parámetros de la concentración crítica de 
a)

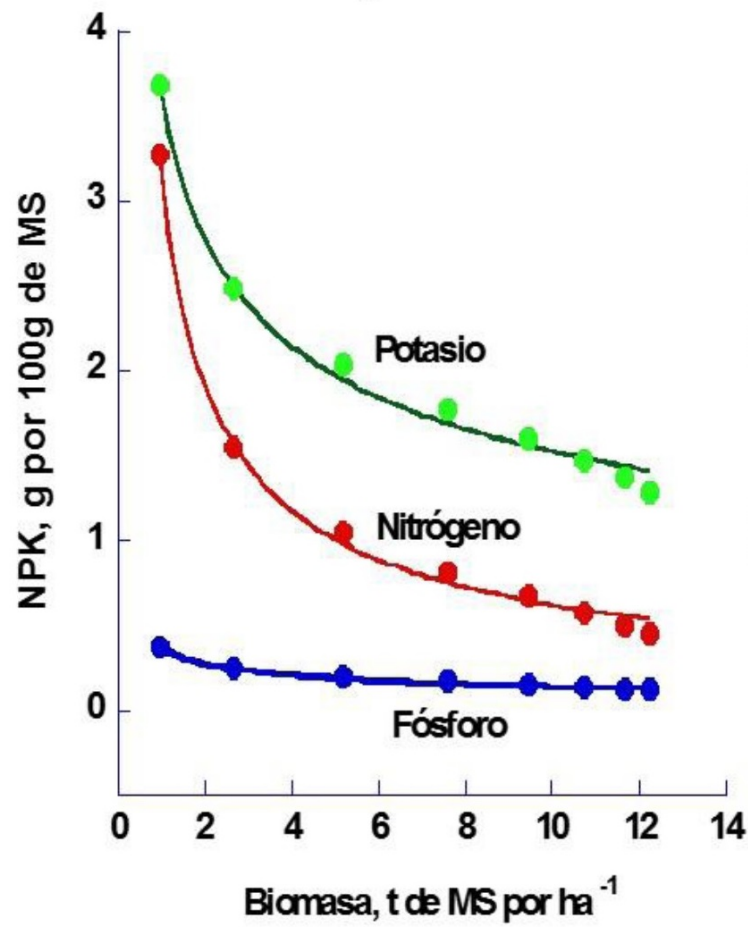

b)

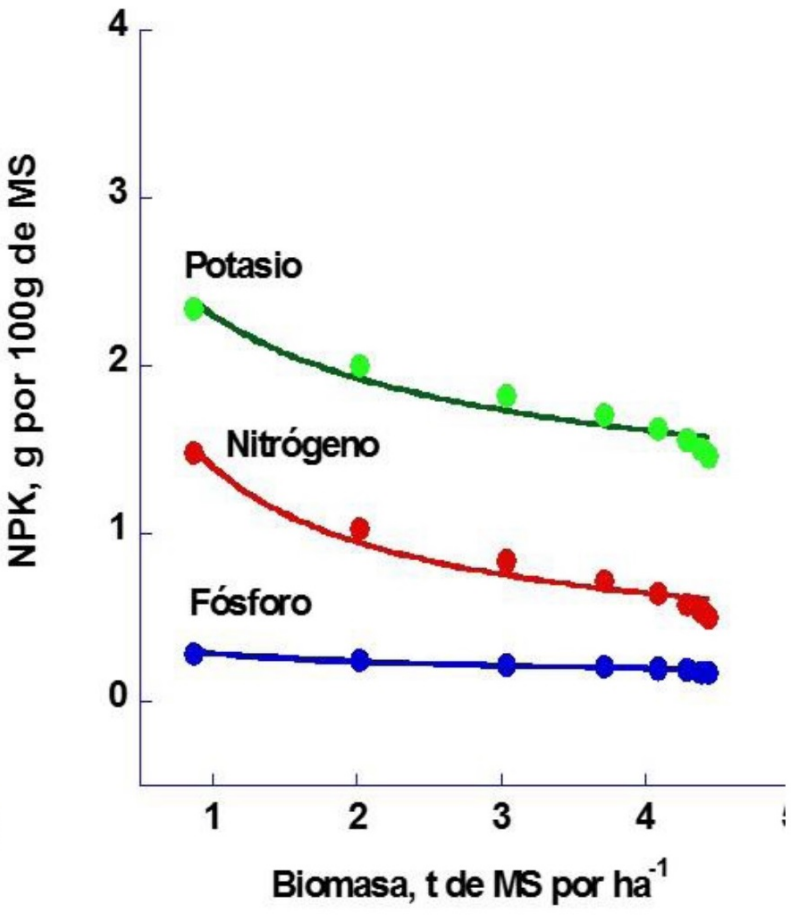

Figura 2. Curvas de los componentes NPK durante el crecimiento del cultivar Taiwán a) Fertilizar b) Sin Fertilizar, ajustados con la ecuación de potencia.

Tabla 3. Parámetros de la concentración crítica y del coeficiente de disminución de los nutrientes NPK del cultivar Taiwán, con y sin fertilización.

\begin{tabular}{lcccccccc}
\hline Variable & \multicolumn{4}{c}{ Fertilizado } & \multicolumn{4}{c}{ Sin fertilizar } \\
& $\mathrm{M}_{1}$ & $\mathrm{EE}$ & $\mathrm{M}_{2}$ & $\mathrm{EE}$ & $\mathrm{M}_{1}$ & $\mathrm{EE}$ & $\mathrm{M}_{2}$ & $\mathrm{EE}$ \\
\hline Nitrógeno & 3.10 & 0.05 & -0.70 & 0.02 & 1.41 & 0.06 & -0.56 & 0.05 \\
Fósforo & 0.37 & 0.01 & -0.41 & 0.01 & 0.29 & 0.01 & -0.27 & 0.03 \\
Potasio & 3.59 & 0.01 & -0.37 & 0.01 & 2.31 & 0.07 & -0.26 & 0.02 \\
\hline $\mathrm{M}_{1}=$ Concentración critica del componente en $\mathrm{g} 100 \mathrm{~g}^{-1} \mathrm{MS}$ para producir una \\
tonelada de MS en su máximo crecimiento; $\mathrm{M}_{2}=$ Coeficiente de la disminución \\
de la concentración de cada nutriente durante el crecimiento EE = Error están- \\
dar.
\end{tabular}

Tabla 4. Bondad de ajuste de las curvas de NPK del cultivar Taiwán durante el crecimiento, fertilizado y sin fertilizar en la época de lluvias en la llanura Veracruzana.

\begin{tabular}{lcccc}
\hline \multirow{2}{*}{ Variable } & \multicolumn{2}{c}{ Fertilizado } & \multicolumn{2}{c}{ Sin fertilizar } \\
& Coeficiente & Chi cuadrada & Coeficiente & Chi cuadrada \\
& correlación, R & $X^{2}$ & correlación, R & $X^{2}$ \\
\hline Nitrógeno & 0.998 & 0.021 & 0.975 & 0.036 \\
Fósforo & 0.996 & 0.0003 & 0.968 & 0.0006 \\
Potasio & 0.996 & 0.035 & 0.967 & 0.039 \\
\hline
\end{tabular}

NPK y el coeficiente de disminución de los nutrientes NPK. Valores muy bajos de $X^{2}$ en el Taiwán fertilizado y no fertilizado, producto de la diferencia de los datos observados y estimados, son resultando de un ajuste con gran certidumbre en la estimación de los parámetros.

Las curvas de extracción de N, P, K, Ca, Mg y $S$ en $\mathrm{kg} \mathrm{ha}^{-1}$, durante el crecimiento del cultivar 
Taiwán, con y sin fertilizar se presentan en la Figura 3. En la mayoría de los casos los datos observados se ajustaron a un polinomio de orden cuadrático, los datos estimados se presentan en forma de gráficos. En la Figura 3, se muestran las curvas de extracción del $\mathrm{N}, \mathrm{P}, \mathrm{K}, \mathrm{Ca}, \mathrm{Mg}$ y $\mathrm{S}$ en $\mathrm{kg} \mathrm{ha}^{-1}$ del Taiwán fertilizado y sin fertilizar, después del ajuste con el polinomio cuadrático. Para un pasto Taiwán fertilizado, la máxima extracción de $\mathrm{N}$ (63 $\mathrm{kg} \mathrm{N}$ ha $^{-1}$ ), es a los 98 días del crecimiento, y su tasa de crecimiento máxima se presenta al inicio de los 77 días de edad. En la curva de extracción de fósforo, esto continua hasta el día 119 de crecimiento, con $15 \mathrm{~kg} \mathrm{ha}^{-1}$ de $\mathrm{P}$ y en el caso del potasio continúa la extracción hasta el día 140 con un valor de $162 \mathrm{~kg} \mathrm{~K}$ ha $^{-1}$, similar comportamiento ocurrió para el $\mathrm{Ca}$, Mg y S con 61, 15 y 1.7 $\mathrm{kg} \mathrm{ha}^{-1}$, respectivamente. Para el caso del Taiwán sin fertilizar, presentó el mismo comportamiento que los fertilizados en cada caso de los nutrientes $\mathrm{N}, \mathrm{P}, \mathrm{K}$, $\mathrm{Ca}, \mathrm{Mg}$ y $\mathrm{S}$ en $\mathrm{kg} \mathrm{ha}^{-1}$.

La Eficiencia del Uso, Absorción y Conversión de NPK aplicado, durante el crecimiento del cultivar Taiwán, En la Figura 4a, que muestra el comportamiento de la Eficiencia del Uso de la aplicación de fertilización de NPK durante el crecimiento del cultivar Taiwán. La eficiencia del uso de nitrógeno proveniente de la aplicación de $141 \mathrm{~kg}_{\text {de }} \mathrm{N} \mathrm{ha}^{-1}$ presenta un comportamiento lineal, es decir, la eficiencia del uso de la aplicación de nitrógeno se incrementa con el tiempo. De forma similar ocurre con la eficiencia del uso del fósforo y potasio, es decir aumenta con el tiempo de crecimiento.

\section{DISCUSIÓN}

Considerando, que el forraje se utilice como corte o pastoreo en la alimentación de bovinos, es importante elegir el tiempo de utilización, con la mejor calidad nutricional, y esto se da, encontrando la máxima tasa de crecimiento (Tabla 1). Para el caso del forraje fertilizado, la tasa máxima se logró entre los días 77-98 de edad y para el forraje sin fertilizar, la tasa máxima de crecimiento ocurrió entre los 42-63 días de rebrote. Si comparamos los valores obtenidos del punto de inflexión con el modelo de Gompertz, se observa que para el forraje fertilizado fue de 54 días y sin fertilizar de 34 días; es decir, este parámetro fue subestimado por el modelo, por lo que es mejor en este caso, emplear los valores de la tasa de crecimiento. Los indicadores de bondad de ajuste permitieron definir cuando un modelo que es aplicado a una serie de datos se ajusta y puede predecir con un grado de confiabilidad elevado, pero esto no indica que los parámetros determinados sean los adecuados, por lo que es preciso verificar la interpretación biológica de esos parámetros (Winsor 1932).

Los datos de Tabla 3, indican que el forraje del cultivar Taiwán en suelos modernamente ácidos y contenido medio de materia orgánica, requieren 186 $\mathrm{kg} \mathrm{N} \mathrm{ha}^{-1}$, para producir $6000 \mathrm{~kg}$ de MS por ha ${ }^{-1}$, de estos el $23.2 \%$ fue extraído del suelo y $142 \mathrm{~kg}$ de $\mathrm{N}$ ha $^{-1}$ fueron absorbidos por efecto de la fertilización. Para es el caso de Fósforo se encontró que requiere $22.5 \mathrm{~kg}$ de $\mathrm{P} \mathrm{ha}^{-1}$, para obtener $6000 \mathrm{~kg}$ de MS por $\mathrm{ha}^{-1}$, del cual, $39.4 \%$ los obtuvo del suelo. Situación diferente se encontró con el Potasio en el forraje, ya que a pesar de que el requerimiento encontrado (218 $\mathrm{kg} \mathrm{K} \mathrm{ha}^{-1}$ ) fue muy superior al aplicado (20 kg de $\mathrm{K}_{2} \mathrm{O} \mathrm{ha}^{-1}$ ), el restante el forraje lo extrajo del suelo. Con relación a lo anterior, con una producción de 28 $200 \mathrm{~kg}$ de $\mathrm{MS} \mathrm{ha}^{-1}$ año $^{-1}$, este pasto requirió extraer del suelo; 338, 72, 564, 108 y $71 \mathrm{~kg} \mathrm{ha}^{-1}$ año ${ }^{-1}$ de $\mathrm{N}, \mathrm{P}, \mathrm{K}, \mathrm{Ca}$ y $\mathrm{Mg}$, respectivamente (Chandler et al. 1983). Estos valores son cercanos a la producción alcanzada en este estudio, con un solo corte a los 77 días de crecimiento.

Los coeficientes de disminución de nutrientes $\mathrm{M}_{2}$ para el Taiwán fertilizado con una producción de $6 \mathrm{t} \mathrm{ha}^{-1}$ para $\mathrm{N}$ fue de $69.8 \%$, P $40.9 \%$ y K $37.3 \%$, a pesar de que los contenidos son altos al inicio del crecimiento, estos se pierden rápidamente, a diferencia del Taiwán sin fertilizar con un crecimiento más lento, los valores son menores (N, 55\%; P, 27\%; y $\mathrm{K}, 25 \%$ ) con una producción de $3 \mathrm{t} \mathrm{ha}{ }^{-1}$. Al respeto, en Brachiaria híbrido para tener una producción de $3 \mathrm{t} \mathrm{ha}^{-1} \mathrm{MS}$ se reporta un coeficiente $\mathrm{M}_{2}$ del $24 \%$ (Juárez y Bolaños 2007). Lo que significa que a un rápido crecimiento hay mayor pérdida o dilución de nutrientes. Los resultados de la aproximación para determinar las necesidades de nutrientes, para producir $6000 \mathrm{~kg}$ de MS por ha ${ }^{-1}$, indican que fueron 
Nitrógeno

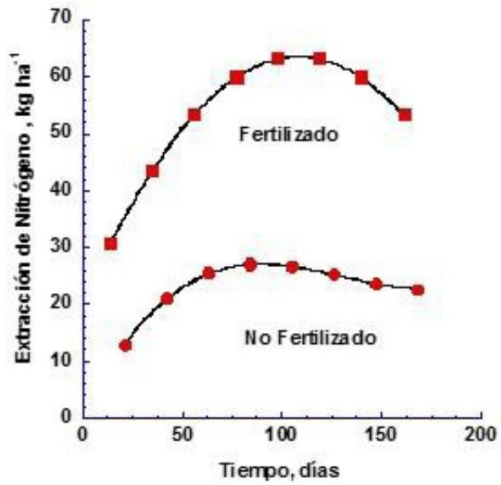

Potasio

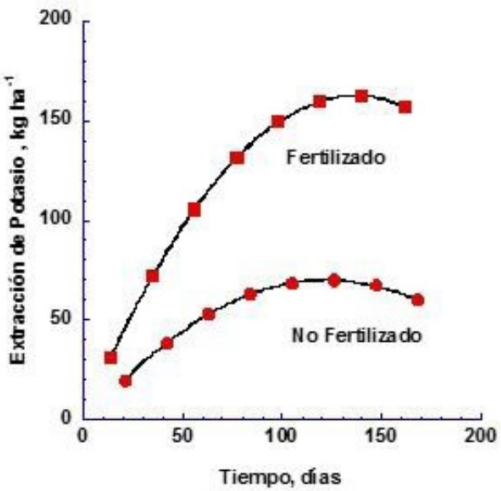

Magnesio

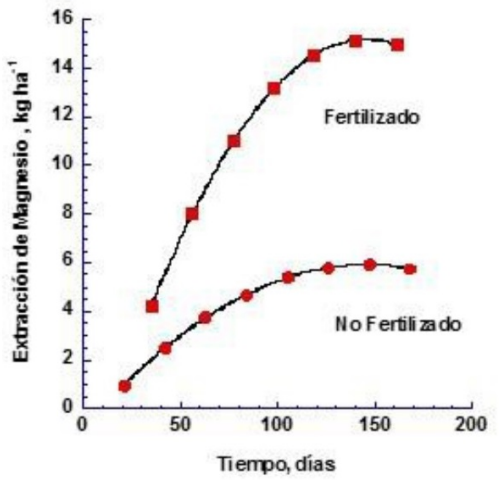

Fósforo

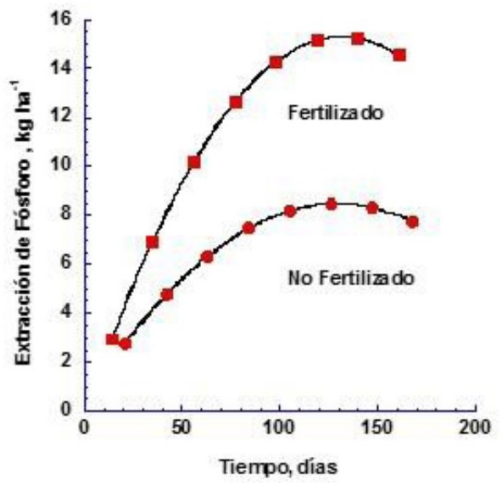

Calcio

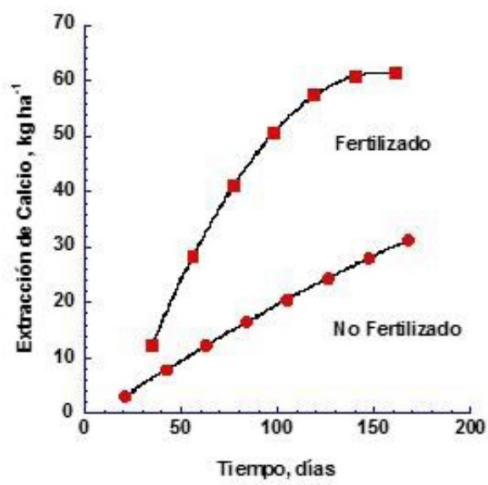

Azufre

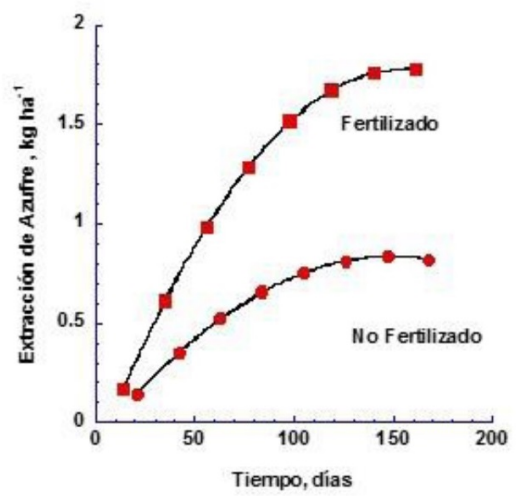

Figura 3. Curvas de extracción de $\mathrm{N}, \mathrm{P}, \mathrm{K}, \mathrm{Ca}, \mathrm{Mg}$ y S en $\mathrm{kg} \mathrm{ha}^{-1}$, durante el crecimiento del cultivar Taiwán con y sin fertilizar. 
a)

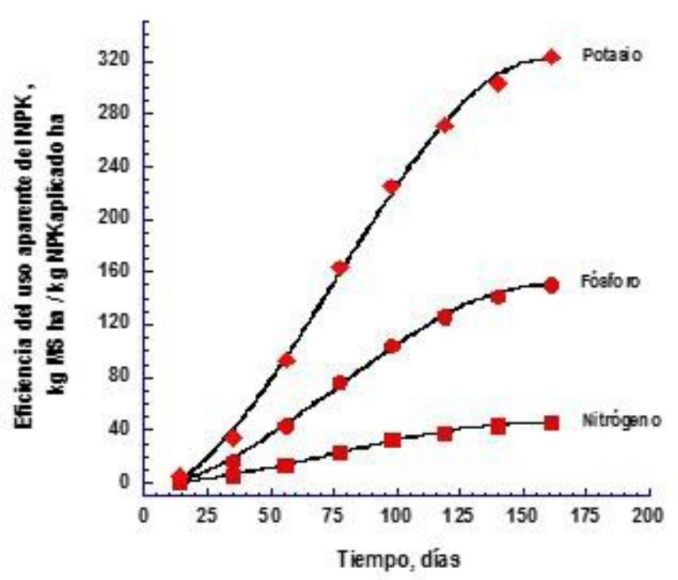

c)

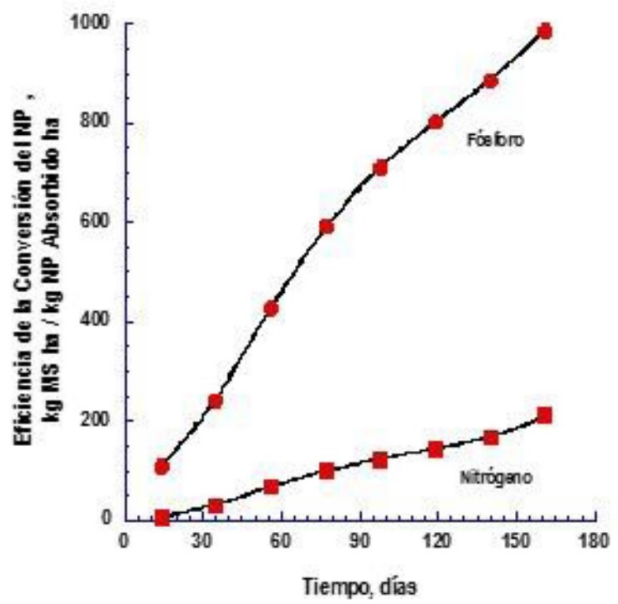

b)

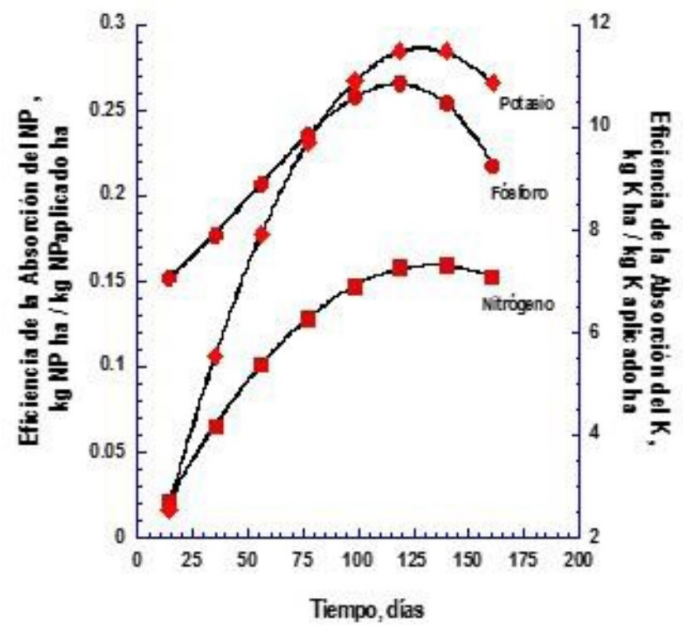

d)

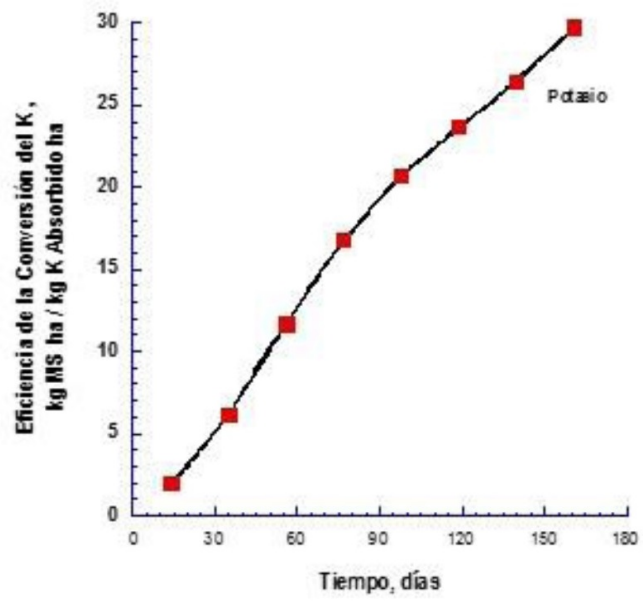

Figura 4. a) Eficiencia del Uso de NPK, b) Eficiencia de la Absorción de NPK, c) Eficiencia de la conversión de NP y d) Eficiencia de la conversión de $\mathrm{K}$ aplicados, durante el crecimiento del cultivar Taiwán.

necesarios $186 \mathrm{~kg} \mathrm{ha}^{-1}$ de $\mathrm{N}$, de los cuales el $30.9 \%$ se obtiene del suelo, lo que indica que el fertilizante aplicado no fue suficiente $\left(141 \mathrm{~kg} \mathrm{ha}^{-1}\right)$. Para el $\mathrm{P}$ fue de $32.7 \mathrm{~kg} \mathrm{ha}^{-1}$, de los cuales, el $42.2 \%$ fue obtenido del suelo. El $\mathrm{K}$ presento una absorción acumulada de $229 \mathrm{~kg} \mathrm{ha}^{-1}$, extraído en un $90 \%$ del suelo, mientras que el resto fue como fertilizante. Aunque el $\mathrm{Ca}$, $\mathrm{Mg}$ y S son aportados en las dosis de fertilización, una tercera parte de ellos fue extraída del suelo y las cantidades requeridas fueron 52,15 y $2 \mathrm{~kg} \mathrm{ha}^{-1}$, respectivamente.

Con respecto a la Eficiencia del Uso, Absorción y Conversión de NPK aplicado, si tomamos la referencia de la tasa máxima de crecimiento a los 77 días, el valor correspondiente equivale a $23 \mathrm{~kg}$ $\mathrm{MS} \mathrm{ha}^{-1}$ por cada $\mathrm{kg}$ de $\mathrm{N}$ aplicado, en el supuesto 
que no existieran pérdidas en el suelo del $\mathrm{N}$ aplicado y que sea los requerimientos establecidos para ese crecimiento en el caso del Taiwán. Al respecto, Marino et al. (2004) reportan valores de 15 y $52 \mathrm{~kg}$

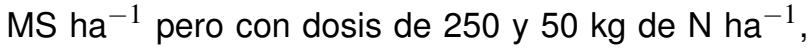
respectivamente, en pasto ryegrass. El $\mathrm{P}$ y el $\mathrm{K}$ presentaron mismo comportamiento con valores de 75 y $172 \mathrm{~kg} \mathrm{MS} \mathrm{ha}^{-1}$ por $\mathrm{kg}$ de $\mathrm{P}$ y K aplicado como fertilizante. En la Figura $4 b$ se describen las tendencias de la Eficiencia de la aplicación de NPK como fertilizante al cultivar Taiwán, sobre la producción de NPK absorbido durante el crecimiento. En todos los casos se observa que su máxima capacidad de absorción por kg de NPK aplicado como fertilizante se presenta, cuando ocurre la máxima producción de biomasa (curva de crecimiento), con 0.254, 0.159 y $11.46 \mathrm{~kg}$ de NPK absorbido respectivamente por kg de NPK aplicado como fertilizante. Jeuffroy y Meynard (1997), Jarvis y Macduff (1989) reportaron la misma tendencia. En las Figura $4 \mathrm{c}$ y d, se presentan las tendencias de la eficiencia de la conversión en biomasa en $\mathrm{kg} \mathrm{MS} \mathrm{ha}^{-1}$, por efecto de $\mathrm{kg}$ NPK absorbido durante el crecimiento. Este comportamiento tiene una tendencia lineal, es decir, se incrementa a través del tiempo. En este caso, es importante tomar como referencia, los días (77) de crecimiento máximo, y al respecto se encontraron valores de 98, 590 y $16 \mathrm{~kg} \mathrm{MS} \mathrm{ha}^{-1}$ convertidos en biomasa por efecto de cada $\mathrm{kg}$ de NPK absorbido durante el crecimiento del Taiwán, es decir, que se encontraron altas producciones de biomasa por cada $\mathrm{kg}$ absorbido de NPK.

\section{CONCLUSIONES}

El cultivar Taiwán en la llanura Veracruzana en donde los suelos son vertisoles moderadamente ácidos requiere $186 \mathrm{~kg} \mathrm{~N}, 27 \mathrm{~kg} \mathrm{P}_{2} \mathrm{O}_{5}, 279 \mathrm{~kg} \mathrm{~K} \mathrm{O}_{2}$, $75 \mathrm{~kg} \mathrm{Ca}, 22 \mathrm{~kg} \mathrm{Mg} \mathrm{y} 3 \mathrm{~kg}$ de S, para producir aproximadamente $6000 \mathrm{~kg}$ de $\mathrm{MS} \mathrm{ha}^{-1}$ en la época de lluvias, con máximo crecimiento a los 77 días de rebrote. Mientras que sin fertilizar, requiere $53 \mathrm{~kg} \mathrm{~N}$, $11 \mathrm{~kg} \mathrm{P}_{2} \mathrm{O}_{5}, 90 \mathrm{~kg} \mathrm{~K} 2 \mathrm{O}, 23 \mathrm{~kg} \mathrm{Ca}, 7 \mathrm{~kg} \mathrm{Mg}$ y $1 \mathrm{~kg}$ de $\mathrm{S}$, para producir aproximadamente $3000 \mathrm{~kg}$ de MS $\mathrm{ha}^{-1}$, con máximo crecimiento entre $42-63$ días de rebrote.

\section{AGRADECIMIENTOS}

Al Dr. Isaías López Guerrero, ex-investigador del Campo Experimental La Posta-INIFAP, quien inició y apoyó esta investigación, así como la evaluación de diversos cultivares de Cenchrus purpureus.

\section{LITERATURA CITADA}

Agnusdei MG, Assuero SG, Lattanzi FA, Marino MA (2010) Critical N concentration can vary with growth conditions in forage grasses: Implications for plant $\mathrm{N}$ status assessment and $\mathrm{N}$ deficiency diagnosis. Nutrient Cycling Agroecosystem 88: 215-230.

Calzada-Marín JM, Enríquez-Quiroz JF, Hernández-Garay A, Ortega-Jiménez E, Mendoza-Pedroza SI (2014) Análisis de crecimiento del pasto maralfalfa (Pennisetum sp.) en clima cálido subhúmedo. Revista Mexicana de Ciencias Pecuarias 5: 247-260.

Chacín F (1998) Análisis de regresión y superficie de respuesta. (ed) Casares/ Segovia / Gaskin. Facultad de Agronomía. Universidad Central de Venezuela. Maracay, Venezuela. 431p.

Chandler JV, Caro-Costas R, Abruña F, Silva S (1983) Producción y utilización intensiva de las forrajeras en Puerto Rico. Universidad de Puerto Rico. Recinto Mayaguez Colegio de Ciencias Agrícolas. Estación Experimental Río Piedras. Boletín 271. Puerto Rico. 228p.

Cook B, Pengelly B, Schultze-Kraft R, Taylor M, Burkart S, Cardoso AJA, González GJJ, Cox K, Jones C, and Peters M (2020) Cenchrus purpureus \& hybrids. Tropical Forages: an interactive selection tool https://tropical grasslands.info/index.php/tgft/pages/view/News. Fecha de consulta: 23 de septiembre de 2021. 
Dreccer MF, Schapendonk AHCM, Slafer GA, Rabbinge R (2000) Comparative response of wheat and oilseed rape to nitrogen supply: Absorption and utilization efficiency of radiation and nitrogen during the reproductive stages determining yield. Plant Soil 220: 189-205.

Fagundes JL, Fonseca DM, Morais RV, Mistura C, Vitor CM, Gomide JA, Nascimento Junior DD, Santos ME, Lambertucci DM (2006) Avaliação das características estructurais do capimbraquiáría em pastagens adubadas com nitrógeno nas quatro estações do ano. Revista Brasileira de Zootecnia 35: 30-37.

Flénet F, Guèrif M, Boiffin J, Dorvillez D, Champolivier L (2006) The critical N dilution curve for linseed (Linum usitatissimum L.) is different from other C3 species. European Journal Agronomy 24: 367-373.

France J, Thornley JMH (1984) Mathematical models in agriculture. A quantitative approach to problems in agriculture and related sciences. Agricultural Systems 18: 65-66.

Horgan GW (2005) Growth curve modeling. Biomathematics and statistics. Scotland. Rowett Institute, University Aberdeen, Foresterhill. Aberdeen Scotland UK. https://www.bioss.ac.uk/training/courses.html. Fecha de consulta: 21 de septiembre 2021.

INEGI (2009) Instituto Nacional de Estadística y Geografía. Catálogo de claves de entidades federativas, municipios y localidades. http://mapserver.inegi.org.mx/mgn2k/?s=geo\&c=1223. Fecha de consulta: 21 septiembre 2021.

Isfan D (1993) Genotype variability for physiological efficiency index of nitrogen in oats. Plant Soil 154: 53.59.

Juárez HJ, Bolaños AED (2007) Las curvas de dilución de la proteína como alternativa para la evaluación de pastos tropicales. Universidad y Ciencia 23: 81-90.

Justes EB, Mary B, Meynard JM, Machet JM, Thelier-Huche L (1994) Determination of a critical nitrogen dilution curve for winter wheat crops. Annals of Botany 74: 397-407.

Jarvis SC, Macduff JH (1989) Nitrate nutrition of grasses from steady-state supplies in flowing solution culture following nitrate deprivation and/or defolation: I. Recovery of uptake and growth and their interactions. Journal Experimental Botany 40: 965-975.

Jeuffroy MH, Meynard JM (1997) Azote: Production agricole et environnement. In: Jean-François, Morot-Gaudry (ed) Assimilation de lázote chez les plantes: Aspects physiologique, biochimique et moléculaire. INRA Paris. pp: 370-380.

Lawrence TLJ, Fowler VR (2002) Prenatal and postnatal growth. Growth of farm animals. $2^{\text {th }}$ editions. CAB International. 347p.

López-Astilleros O, Vinay-Vadillo JC, Villegas-Aparicio Y, López Guerrero I, Lozano-Trejo S (2020) Dinámica de crecimiento y curvas de extracción de nutrientes de Pennisetum sp. (Maralfalfa). Revista mexicana de ciencias pecuarias.11: 255-265.

Marino MA, Mazzanti A, Assuero SG, Gastal F, Echeverría HE, Andrade F (2004) Nitrogen dilution curves and nitrogen use efficiency during winter-spring growth of annual ryegrass. Agronomy Journal 96: 601-607.

Negawo AT, Teshome A, Kumar A, Hanson J, Jones CS (2017) Opportunities for Napier grass (Pennisetum purpureum) improvement using molecular genetics. Agronomy 7(2): 28. DOI: 10.3390/agronomy7020028

Ortega RL, Enríquez JFQ, López GI (2009) Producción sustentable de forrajes tropicales. En: Román PH, Ortega LR, Hernández LA, Díaz EA, Espinosa JAG, Núñez GH, Vera RA, Medina MC, Ruiz FJL (Comp.) Producción de leche de bovino en el sistema de doble propósito. Libro Técnico Núm. 22. INIFAP. CIRGOC. Veracruz, México. pp: 19-49. 
Ortiz-Monasterio JI, Sayre RKD, Rajaram S, McMahon M (1997) Genetic progress in wheat yield and nitrogen use efficiency under four plant material. Crop Science 37: 898-904.

Raji AO, Alade NK, Duwa H (2014) Estimation of model parameters of the Japanese quail growth curve using Gompertz model. Archivos de zootecnia 63: 429-35.

Ramírez JL, Herrera RS, Leonard I, Cisneros M, Vardecia D, Álvarez Y (2011) Relación entre factores climáticos, rendimiento y calidad de Pennisetum purpureum vc. Cuba CT 169 en el Valle del Cauto, Cuba. Revista Cubana Ciencias Agrícolas 45: 293-297.

Ramos-Sánchez A (1985) Logros y aportaciones de la investigación agrícola en el cultivo de forrajes zonas tropicales y subtropicales. Secretaria de Agricultura y Recursos Hidráulicos, Instituto Nacional de Investigaciones Agrícolas. Publicación especial. México. pp: 117: 5-30.

Reyes-Castro S, Enríquez-Quiroz JF, Hernández-Garay AC, Esqueda-Esquivel VA, Gutiérrez-Arenas DA (2018) Rendimiento de seis cultivares de Cenchrus purpureus (Schumach.) Morrone con potencial para producción de bioetanol. Agroproductividad 11: 56-62.

Rodríguez L, Torres V, Martínez RO, Jay O, Noda AC, Herrera M (2011) Modelos para estimar la dinámica de crecimiento de Pennisetum purpureum vc. Cuba CT-169. Revista cubana de ciencia agrícola 45: 349-54.

Rueda JA, Ortega-Jiménez E, Hernández-Garay A, Enríquez-Quiroz JF, Guerrero-Rodríguez JD, Quero-Carrillo AR (2016) Growth, yield, fiber content and lodging resistance in eight varieties of Cenchrus purpureus (Schumach.) Morrone intended as energy crop. Biomass and Bioenergy 88: 59-65

Thornley JHM, France J (2007) Growth functions. In: Thornley JHM, France J (eds). Mathematical models in agriculture. Quantitative methods for the plant, animal and ecological sciences. 2nd Ed. printed in the UK by Cromwell Press, Trowbrigde. pp: 136-171.

Turner Jr. ME, Bradley Jr. EL, Kirk KA, Pruitt KM (1976) A theory of growth. Mathematical Biosciences 29: 367-373.

Ventura RJ, Honorato SJA, Hernández GA, Aburto AJA, Vaquera HH, Enríquez QJF (2017) Composición química y rendimiento de biomasa de maralfalfa para producción de etanol de segunda generación. Revista Mexicana De Ciencias Agrícolas 8: 213-219.

Vidal ZR (2005) Las regiones climáticas de México. Temas selectos de Geografía de México. Instituto de Geografía UNAM, México. 144p.

Winsor CP (1932) Gompertz curve as a growth. Proceeding National Academy Science 18: 1-7.

Wilkins PW, Macduff JH, Raistrick N, Collison M (1997) Varietal differences in perennial ryegrass for nitrogen use efficiency in leaf growth following defoliation: Performance in flowing solution culture and its relationship to yield under simulated grazing in the field. Euphytica 98: 109-119.

Ziadi N, Brassard M, Bélanger G, Cambouris AN, Tremblay N, Nolin MC, Claessens A, Parent LÉ (2008) Critical nitrogen curve and nitrogen nutrition index for corn in eastern Canada. Agronomy Journal 100: 271-276. 